\title{
Precisión diagnóstica de la radiografía para la medición de túneles óseos en reconstrucción de ligamento cruzado anterior
}

\section{$X$ Ray Diagnostic Accuracy for Bone Tunnel Measurements in Anterior Cruciate Ligament Reconstruction}

\author{
Juanjosé Valderrama Ronco ${ }^{1}$ Xabier Carredano González ${ }^{1} \quad$ Ignacio Valderrama Sagasti ${ }^{1}$ \\ Diego Montenegro Bralic ${ }^{1}$ Agustín León Iglesias ${ }^{1}$ Gonzalo Ferrer Aguayo ${ }^{1}$ Gonzalo Espinoza Lavín ${ }^{1}$
${ }^{1}$ Traumatología, Hospital Clínico de la Mutual de Seguridad, Santiago, Address for correspondence Juanjose Valderrama Ronco, MD, Chile Traumatología, Hospital Clínico de la Mutual de Seguridad, Santiago, Chile (e-mail: juanjovalderrama@gmail.com). Rev Chil Ortop Traumatol 2019;60:3-8.

\begin{abstract}
Resumen
Palabras Clave

- reconstrucción LCA

- túneles

- diámetros

- Rx

$-\mathrm{TC}$

Objetivo Determinar la precisión diagnóstica de las radiografías de rodilla para la medición de los diámetros de los túneles tibiales y femorales en reconstrucción de ligamento cruzado anterior (RLCA).

Materiales y Pacientes Estudio retrospectivo de precisión diagnóstica en pacientes con antecedentes de RLCA. Inclusión: RLCA realizada en el mismo centro, con el mismo equipo quirúrgico, entre 2011 y 2015; uso de cualquier tipo de injerto y de fijación; estudiados con radiografía ( $\mathrm{Rx}$ ) y tomografía computada (TC) durante postop en el mismo centro. Exclusión: desfase Rx-TC > 6 meses; cirugía entre estudios radiológicos. Análisis de túneles por 3 especialistas (OsiriX). La precisión diagnóstica consideró la TC como gold standard. Se midieron las diferencias inter e intra-observador, y las variables que interfirieron en las mediciones.

Resultados 22 pacientes cumplieron los criterios de selección, determinando 528 mediciones en total (Rx y TC, fémur y tibia, 3 observadores, 2 tiempos). No hubo diferencias estadísticamente significativas en la medición de los túneles femorales $(p=0.8986)$, pero sí en cuanto a los diámetros tibiales $(p=0.0001)$. El análisis de precisión diagnóstica determinó una sobrevaloración de los diámetros óseos al usar la radiografía (10,5\% en fémur, $10 \%$ en tibia). Hubo diferencias estadísticamente significativas inter-observador tanto en Rx como en TC (observador más joven con el resto); sin diferencias intra-observador.

Conclusión La Rx como método diagnóstico del diámetro de túneles óseos luego de una RLCA sobreestima los valores reales, lo que empeora cuando el observador tiene menor experiencia.
\end{abstract}

Abstract
Objective To determine the diagnostic accuracy of knee radiographs for the measurement of tibial and femoral tunnels diameters after an anterior cruciate ligament reconstruction (ACLR). received

September 20, 2018

accepted

February 13, 2019
DOI https://doi.org/

$10.1055 / \mathrm{s}-0039-1683989$

ISSN $0716-4548$.
Copyright $\odot 2019$ by Thieme Revinter

Publicações Ltda, Rio de Janeiro, Brazil

License terms

c) $(1) \$$ 


\author{
Keywords \\ - $\mathrm{ACL}$ reconstruction \\ - tunnels \\ - diameter \\ - X ray \\ $-\mathrm{CT}$
}

\begin{abstract}
Materials and Patients A retrospective study of the diagnostic accuracy in patients with a history of ACLR. Inclusion: surgery performed in the same center, same surgical team, between 2011 to 2015; any graft and fixation; studied with radiography (X-Ray) and computed tomography (CT) during postop in the same center. Exclusion: X-Ray-CT lag $>6$ months; surgery between radiological studies. Tunnel analysis by 3 specialists (OsiriX). Diagnostic accuracy considered CT as a gold standard. The inter and intraobserver differences, and the variables that interfered in the measurements, were measured.

Results 22 patients achieved the selection criteria, determining 528 measurements in total (X-Ray and CT, femur and tibia, 3 observers, 2 times). There were no statistically significant differences in the measurement of the femoral tunnels $(p=0.8986)$, but there were differences in the tibial diameters $(p=0.0001)$. Analysis of diagnostic accuracy determined an overestimation of the bone diameters when using the radiography $(10.5 \%$ in the femur, $10 \%$ in the tibia). There were statistically significant inter-observer differences in both X-Ray and CT (younger observer with the rest); there were no intra-observer differences.

Conclusion X-Ray as a diagnostic method of the diameter of bone tunnels after an ACLR overestimates the real values, which worsens when the observer has less experience.
\end{abstract}

\section{Introducción}

La rotura del ligamento cruzado anterior (LCA), corresponde a una de las lesiones ligamentarias más comunes en la rodilla, la incidencia estimada de esa lesión en Estados Unidos al año 2006 fue de 43,5 lesiones cada 100,000 personas/año. ${ }^{1}$ Su reconstrucción determina buenos resultados; sin embargo, en ocasiones, y debido a una mala técnica quirúrgica, rehabilitación inadecuada o a un nuevo trauma, puede terminar con resultados insatisfactorios. ${ }^{2}$

Posterior a una reconstrucción de LCA (RLCA), pueden ocurrir fenómenos de ensanchamiento de los túneles óseos, tanto en el fémur como en la tibia. Su etiología, los efectos sobre el injerto y las dificultades que genera en las cirugías de revisión han sido descrita por múltiples autores. ${ }^{3-8}$ Ese hecho sucede predominantemente durante los primeros 6 meses post operatorios, momento en que se establece la interfaz entre el tendón y el hueso; sin embargo, su aparición ha sido descrito incluso a los 2 años post quirúrgicos, dando cuenta de una etiología probablemente multifactorial y una magnitud variable. , $^{3,8}$

Si bien la implicancia del ensanchamiento de los túneles óseos sobre la falla de la reconstrucción, la inestabilidad residual o el desarrollo de artrosis no se ha logrado comprender a cabalidad, su mayor aplicación clínica radica en la planificación de las cirugías de revisión de LCA, donde es imprescindible conocer con la mayor precisión posible, el diámetro de los túneles para una mejor planificación de los insumos requeridos, la técnica quirúrgica a utilizar ( $\begin{array}{lll}1 & 0 & 2\end{array}$ tiempos) y el tipo de injerto. ${ }^{3}$

Los estudios iniciales del ensanchamiento fueron realizados con radiografías $(\mathrm{Rx})^{9-11}$; no obstante, estudios posteriores han encontrado discrepancias en cuanto a las mediciones de los diámetros de los túneles entre Rx, tomografías computadas (TC) y Resonancias Magnéticas
$(\mathrm{RM})^{12,13}$; sin embargo, según el estudio de Marchant y col., la TC tendría claras ventajas sobre los otros dos métodos diagnósticos. ${ }^{3}$

Actualmente, pese a la evidencia disponible en cuanto a la precisión de los diferentes estudios imagenológicos, hay publicaciones que continúan utilizando las radiografías como método diagnóstico para la medición del diámetro de los túneles. ${ }^{14-16}$ Además, si bien existen algunos estudios que sugieren la TC como la herramienta diagnóstica que aporta mayor precisión en cuanto a las mediciones ya descritas; ellos carecen de una metodología estricta y de modelos matemáticos adecuados.

El objetivo principal de este estudio, fue determinar la precisión diagnóstica de las radiografías para la medición de los diámetros de los túneles femorales y tibiales luego de una reconstrucción de LCA.

Consideramos como hipótesis que la $\mathrm{Rx}$ tiene una inadecuada precisión diagnóstica para la medición de los diámetros femorales y tibiales en comparación con la TC.

\section{Materiales y Pacientes}

Se realizó un estudio observacional analítico diagnóstico de temporalidad transversal en donde se midieron los diámetros de los túneles femorales y tibiales en pacientes con antecedentes de RLCA determinando las características generales de esos diámetros y sus diferencias en cuanto a las mediciones en Rx versus TC.

Se consideraron los siguientes criterios inclusión para los casos a estudiar:

- RLCA realizada en el mismo centro hospitalario.

- Mismo equipo quirúrgico.

- Entre los años 2011 y 2015 (año de inicio del registro electrónico de imágenes). 
- Uso de cualquier tipo de injerto y de fijación.

- Estudio imagenológico post operatorio completo, Rx (AP y lat) y TC (solicitado por médico tratante en algún momento de la evolución post operatoria).

- Imágenes tomadas en el mismo centro y en formato DICOM.

Por su parte, los criterios de exclusión fueron:

- Desfase entre Rx y TC >6 meses.

- Cirugías realizadas en la rodilla afectada entre los estudios radiológicos.

Tras la selección de los casos de acuerdo a los criterios ya especificados, se determinó encriptar y aleatorizar todas las imágenes por el autor principal, tanto los estudios imagenológicos con $\mathrm{Rx}$ como con $\mathrm{TC}$, tanto para las mediciones en el tiempo 1 como en el tiempo 2.

Arbitrariamente, se consideraron 3 cirujanos especialistas en rodilla con distintos años de experiencias $(25,10$ y 5 años aproximadamente), para el cálculo de los diámetros.
Cada uno de los cirujanos recibió la misma instrucción para la medición:

- Uso del software OsiriX para el análisis de las distancias.

- Medición y registro del diámetro de mayor amplitud en fémur y en tibia, independiente del plano de análisis, para cada caso imagenológico ( $\mathbf{- F i g . ~ 1 ) . ~}$

- Registro de los datos manualmente en una tabla impresa con los códigos de encriptado de cada imagen (-Fig. 2).

- Repetición de ese método tras al menos 1 semana de la primera medición.

Luego de registrar los datos, se planificó que éstos serían desencriptados y analizados por el autor principal. Se consideró a la TC como siendo el estándar de oro para realizar el cálculo de precisión diagnóstica de las Rx.

Para el análisis estadístico de las diferencias inter e intraobservador, se estimaron las pruebas de la varianza para determinar diferencias, considerando diferencias estadísticamente significativas aquellas con un p-valor $<0,05$. También se valoraron y analizaron otras variables que pudiesen

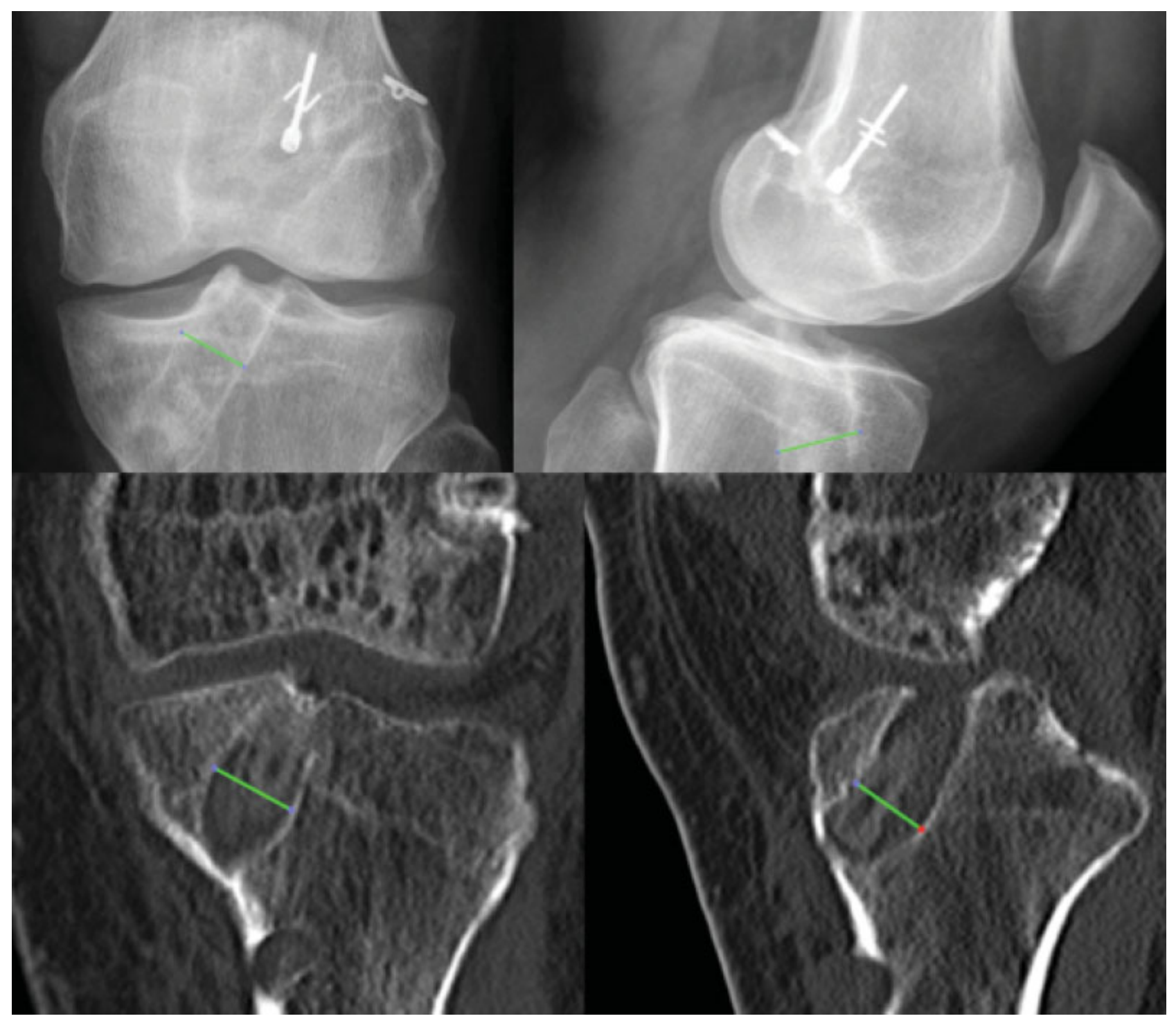

Fig. 1 Mediciones imagenológicas de diámetros en túneles. 
6 Precisión Diagnóstica de Radiografía en Túneles LCA Ronco y col
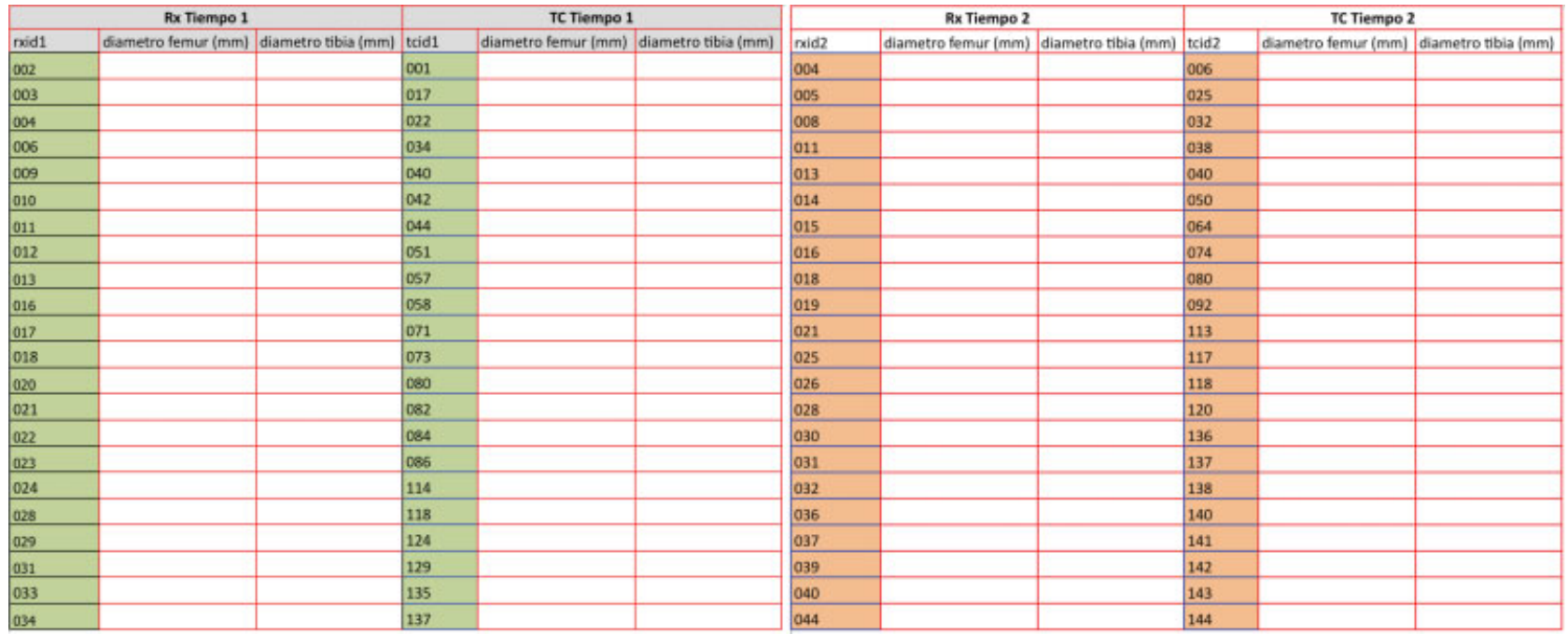

Fig. 2 Tablas de registro tiempo 1 y tiempo 2, datos encriptados.

haber interferido en las mediciones, utilizando el test de correlación de Pearson y regresión lineal simple.

Se determinó realizar un estudio de tamaño muestral post-hoc con un error alfa de 0,05 y una potencia de $80 \%$ para determinar la validez de las mediciones.

\section{Resultados}

Se encontraron 34 pacientes que cumplieron con los criterios de inclusión. De éstos, 8 casos presentaban un desfase entre Rx y TC mayor a 6 meses por lo que se excluyeron. Otros 4 pacientes

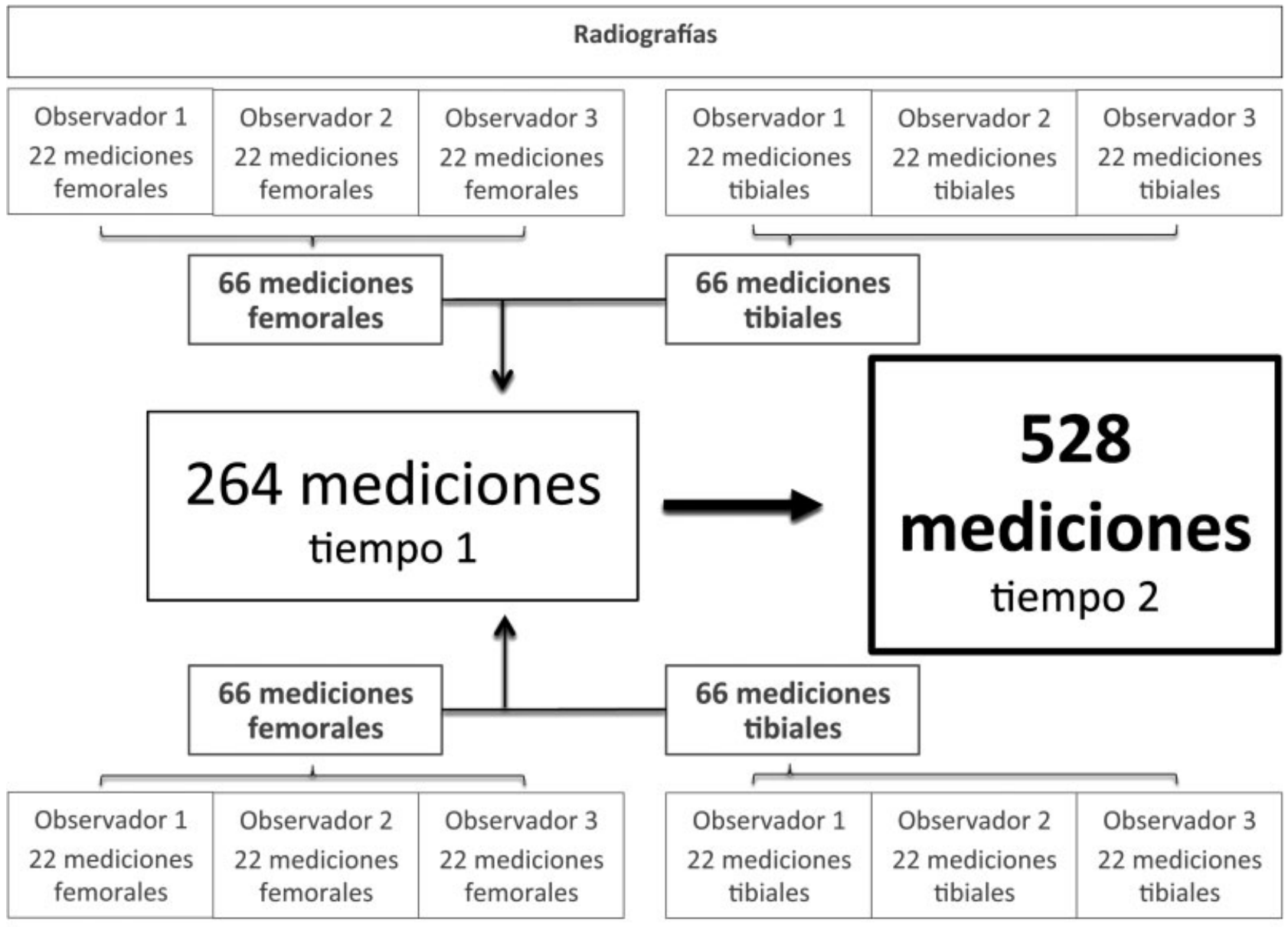

Tomografías Computadas

Fig. 3 Incorporada. 
registraron cirugías entre los estudios imagenológicos por lo que también fueron excluidos, quedando 22 casos.

El total de mediciones realizadas fueron de 528 , las cuales se desglosaron en 22 mediciones femorales y 22 mediciones tibiales radiográficas y tomográficas, tanto para el tiempo 1 como para el tiempo 2, todas realizadas por 3 observadores (-Fig. 3).

El estudio de tamaño muestral post-hoc realizado con la herramienta simple size, determinó un número mínimo de 251 mediciones de fémur en Rx y en TC (hubo 264 mediciones en total); mientras que en tibia resultó de un mínimo de 50 mediciones en $\mathrm{Rx}$ y en TC (hubo 264 mediciones en total), considerando un error alfa de 0,05 y una potencia del $80 \%$.

El promedio de los diámetros se muestran en la - Tabla 1:

Se evidenció que en términos globales de todos los registros, las diferencias de las mediciones en diámetros femorales no fueron estadísticamente significativas, a diferencia de lo que se observó en los diámetros tibiales, en dónde sí las hubo.

De acuerdo al modelo de tendencias de Bland \& Altman, ${ }^{17}$ la precisión diagnóstica para la radiografía, considerando todas los observaciones, fue de sobrevalorar el diámetro de los túneles óseos, lo que se muestra en la -Tabla 2:

En relación al análisis inter observadores utilizando un análisis de varianza, se encontró que no hubo diferencias entre el cirujano de 25 años en comparación con el de 10 años de experiencia. Por su parte, el cirujano con 5 años de experiencia, presentó diferencias estadísticamente significativas en cuanto al uso de Rx en fémur y al uso de TC tanto en fémur como en tibia respecto a los otros dos cirujanos.

El análisis intra observador no arrojó diferencias estadísticamente significativas entre los mismos cirujanos al realizar un análisis de la varianza.

En cuanto al análisis de variables que podría haber influido en ciertas mediciones, se encontró que de 528 mediciones, los cirujanos en términos globales, consideraron que 28 mediciones no fueron posibles de medir por la dificultad técnica que eso significaba (el observador declaró que la imagen era tan poco clara, pese a cambiar contrastes, que no fue posible darle un valor al diámetro). De éstas, el 100\% correspondieron a mediciones

Tabla 1 Diámetros promedios en Rx y TC

\begin{tabular}{|l|l|l|l|}
\hline & Rx & TC & $p$-valor \\
\hline $\begin{array}{l}\text { Túnel } \\
\text { Femoral }\end{array}$ & $\begin{array}{l}10,8 \mathrm{~mm} \\
(8-20, \text { DE 1,8) }\end{array}$ & $\begin{array}{l}10.4 \mathrm{~mm} \\
(8-19, \text { DE 1,8) }\end{array}$ & $\mathbf{0 , 8 9 8 6}$ \\
\hline Túnel & $14 \mathrm{~mm}$ & $12.8 \mathrm{~mm}$ & $\mathbf{0 , 0 0 0 1}$ \\
Tibial & $(10-19$, DE 1,9) & $(8-25$, DE 2,8) & \\
\hline
\end{tabular}

Tabla 2 Precisión de la Radiografía según modelo de Bland \& Altman

\begin{tabular}{|l|l|l|}
\hline Rx & Túneles Femorales & Túneles Tibiales \\
\hline Precisión & 10,5\% Sobreestimación & $10 \%$ Sobreestimación \\
Diagnóstica & (IC 95\% 3.864-17.185) & (IC 95\% 3.653-16.245) \\
\hline
\end{tabular}

en $\mathrm{Rx}, \mathrm{y}$ el 95\% correspondieron a $\mathrm{Rx}$ tomadas antes de las 6 semanas post operatorias.

\section{Discusión}

El ensanchamiento de túneles óseos tras una reconstrucción de LCA es un hecho que sucede, su etiología es considerada multifactorial, dividiéndose en factores mecánicos y biológicos. No obstante la dificultad para determinar su causalidad, la mayor importancia radica en aquellos casos en donde se requiere realizar una cirugía de revisión, puesto que ensanchamientos muy amplios sugieren un manejo en dos tiempos. $^{3-8}$

El uso de radiografías para la medición de ese fenómeno ha sido desacreditada; sin embargo, autores importantes siguen utilizando ese método diagnóstico en publicaciones recientes. $^{14-16}$

Hasta la fecha, no hay estudios indexados que valoren la precisión diagnóstica de la $\mathrm{Rx}$ para el estudio del ensanchamiento de los túneles.

El presente estudio permitió determinar que la precisión diagnóstica de la Rx en comparación con la TC, tiende a sobrevalorar los diámetros en un $10,5 \%$ en los túneles femorales, y en un $10 \%$ en los tibiales, cifras que eventualmente podrían determinar cambios en la conducta quirúrgica.

En cuanto al análisis realizado interobservador, se infiere que la experiencia del cirujano especialista es relevante, razón por la cual es posible que esas mediciones deban ser realizadas en grupo o con la supervisión de un cirujano mediana y/o altamente experimentado.

Por su parte, al no haber diferencias intraobservador en ninguno de los casos, no sería necesario repetir las mediciones, lo cual mejora la confiabilidad de las mediciones grupales o supervisadas como ya fue discutido en el párrafo anterior.

Resulta de suma importancia, considerar la dificultad técnica e imprecisión de las Rx para medir diámetros de túneles óseos antes de las 6 semanas del post operatorio, en caso de ser necesario, con mayor razón se necesitaría el uso de la TC.

Cabe destacar que, en base a los resultados obtenidos, la validez de las mediciones en túneles femorales no fue concluyente, dado la muestra insuficiente para determinar diferencias estadísticamente significativas. No obstante, en las mediciones de los túneles tibiales, el tamaño muestral fue satisfactorio para validar los resultados con una potencia y error alfa adecuados.

\section{Conclusión}

El uso de Rx para la medición de diámetros de túneles óseos, requiere considerar la sobrevaloración de ese método diagnóstico, considerando una precisión limitada principalmente en los túneles tibiales. El cálculo de esas distancias debe hacerse con TC y bajo supervisión de cirujanos con experiencia considerable.

Conflictos de interés

Ninguno 


\section{Bibliografía}

1 Mall NA, Chalmers PN, Moric M, et al. Incidence and trends of anterior cruciate ligament reconstruction in the United States. Am J Sports Med 2014;42(10):2363-2370

2 Wiggins AJ, Grandhi RK, Schneider DK, Stanfield D, Webster KE, Myer GD. Risk of Secondary Injury in Younger Athletes After Anterior Cruciate Ligament Reconstruction: A Systematic Review and Meta-analysis. Am J Sports Med 2016;44(07):1861-1876

3 Marchant MH Jr, Willimon SC, Vinson E, Pietrobon R, Garrett WE, Higgins LD. Comparison of plain radiography, computed tomography, and magnetic resonance imaging in the evaluation of bone tunnel widening after anterior cruciate ligament reconstruction. Knee Surg Sports Traumatol Arthrosc 2010;18 (08):1059-1064

4 Barber FA, Spruill B, Sheluga M. The effect of outlet fixation on tunnel widening. Arthroscopy 2003;19(05):485-492

5 Clatworthy MG, Annear P, Bulow JU, Bartlett RJ. Tunnel widening in anterior cruciate ligament reconstruction: a prospective evaluation of hamstring and patella tendon grafts. Knee Surg Sports Traumatol Arthrosc 1999;7(03):138-145

6 Höher J, Möller HD, Fu FH. Bone tunnel enlargement after anterior cruciate ligament reconstruction: fact or fiction? Knee Surg Sports Traumatol Arthrosc 1998;6(04):231-240

7 Peyrache MD, Djian P, Christel P, Witvoet J. Tibial tunnel enlargement after anterior cruciate ligament reconstruction by autogenous bone-patellar tendon-bone graft. Knee Surg Sports Traumatol Arthrosc 1996;4(01):2-8

8 Aga C, Wilson KJ, Johansen S, Dornan G, La Prade RF, Engebretsen L. Tunnel widening in single- versus double-bundle anterior cruciate ligament reconstructed knees. Knee Surg Sports Traumatol Arthrosc 2017;25(04):1316-1327
9 Barber FA, Elrod BF, McGuire DA, Paulos LE. Preliminary results of an absorbable interference screw. Arthroscopy 1995;11(05):537-548

10 Matthews LS, Parks BG, Sabbagh RC. Determination of fixation strength of large-diameter interference screws. Arthroscopy 1998;14(01):70-74

11 Uribe JW, Hechtman KS, Zvijac JE, Tjin-A-Tsoi EW. Revision anterior cruciate ligament surgery: experience from Miami. Clin Orthop Relat Res 1996;(325):91-99

12 Parkar AP, Adriaensen ME, Fischer-Bredenbeck C, et al Measurements of tunnel placements after anterior cruciate ligament reconstruction-A comparison between CT, radiographs and MRI. Knee 2015;22(06):574-579

13 Webster KE, Feller JA, Elliott J, Hutchison A, Payne R. A comparison of bone tunnel measurements made using computed tomography and digital plain radiography after anterior cruciate ligament reconstruction. Arthroscopy 2004;20(09):946-950

14 Lubowitz JH, Schwartzberg R, Smith P. Cortical Suspensory Button Versus Aperture Interference Screw Fixation for Knee Anterior Cruciate Ligament Soft-Tissue Allograft: A Prospective, Randomized Controlled Trial. Arthroscopy 2015;31(09):1733-1739

15 Group M; MARS Group. Radiographic findings in revision anterior cruciate ligament reconstructions from the Mars cohort. J Knee Surg 2013;26(04):239-247

16 Choi NH, Yang BS, Victoroff BN. Clinical and Radiological Outcomes After Hamstring Anterior Cruciate Ligament Reconstructions: Comparison Between Fixed-Loop and Adjustable-Loop Cortical Suspension Devices. Am J Sports Med 2017;45(04):826-831

17 Bland JM, Altman DG; JM B. Agreement between methods of measurement with multiple observations per individual. J Biopharm Stat 2007;17(04):571-582 